\title{
Isolated monocytosis was the flag preceding abnormalities in other parameters of complete blood counts in chronic myeloid leukemia with e1a2 (minor, P190) BCR-ABL1 chimeric transcripts
}

\author{
Jae Won Yun ${ }^{1}$, Jung Yoon ${ }^{1}$, Jong Won Kim ${ }^{1}$, Sun-Hee Kim ${ }^{1}$, Chul Won Jung ${ }^{2}$, \\ Hee-Jin Kim ${ }^{1}$
}

\begin{abstract}
${ }^{1}$ Department of Laboratory Medicine and Genetics, Samsung Medical Center, Sungkyunkwan University School of Medicine, Seoul, Korea

${ }^{2}$ Division of Hematology-Oncology, Department of Medicine, Samsung Medical Center, Sungkyunkwan University School of Medicine, Seoul, Korea
\end{abstract}

Received: March 14, 2019

Revised: March 18, 2019

Accepted: March 21, 2019

Corresponding author:

Hee-Jin Kim

Department of Laboratory

Medicine and Genetics,

Samsung Medical Center,

Sungkyunkwan University

School of Medicine, 81 Irwon-

ro, Gangnam-gu, Seoul 06351 ,

Korea

Tel: +82-2-3410-2746

E-mail: heejinkim@skku.edu

\begin{abstract}
Chronic myeloid leukemia (CML) is a myeloproliferative neoplasm with the molecular hallmark of $B C R-A B L 1$ chimeric transcripts from $t(9 ; 22)(q 24 ; q 22)$. In more than $95 \%$ of $\mathrm{CML}$, the fusion occurs in the major breakpoint cluster region (M-bcr) of $B C R$, most commonly producing the e14a2 or e13a2 type. CML with e1a2 BCR-ABL1 by fusion in minor bcr is rare, accounting for approximately $1 \%$ of CML. CML with e1a2 BCR-ABL1 is reportedly associated with monocytosis and poor prognosis. Here we describe a woman who underwent bone marrow (BM) study with an impression of chronic myelomonocytic leukemia based on thrombocytosis and prominent monocytosis. Genetic workup revealed, however, $\mathrm{t}(9 ; 22)$ and ela2 BCR-ABL1, which indicated $C M L$ and explained her unusual monocytosis. A review of serial complete blood counts (CBC) before the BM study demonstrated that isolated monocytosis preceded the abnormalities in other parameters of CBC. The patient is on follow-up with tyrosine kinase inhibitor (TKI, dasatinib) medication. Close monitoring is required due to the association of ela2 BCR-ABL1 with poor response to TKI and frequent disease progression. Timely genetic workup and determination of the precise type of $B C R-A B L 1$ transcripts are critical for the diagnosis and stratification of this rare subtype of CML with distinct genotype-phenotype correlations.
\end{abstract}

Keywords: ela2 BCR-ABL1; Leukemia, myelogenous, chronic, BCR-ABL positive; Monocytosis

\section{INTRODUCTION}

Chronic myeloid leukemia (CML) is one of the disease entities included in myeloproliferative
This is an Open Access article distributed under the terms of the Creative Commons Attribution Non-Commercial License (http:// creativecommons.org/licenses/ by-nc/4.0/). 
neoplasms (MPNs) by the 'WHO classification of tumours of haematopoietic and lymphoid tissues' [1]. The BCR-ABL1 chimeric transcripts from the chromosomal translocation $\mathrm{t}(9 ; 22)$ (q34.1;q11.2) is the molecular hallmark of CML and is the therapeutic target of tyrosine kinase inhibitors (TKIs) [2]. Depending on the breakpoints within the breakpoint cluster region gene $(B C R)$ on 22q11 and the Abelson murine leukemia viral oncogene homolog 1 gene ( $A B L 1$ ) on 9q34, various forms of fusion transcripts have been identified [3]. In particular, three representative bcr have been described in the $B C R$ gene: the major bcr ( $M-b c r ;$ the area between exons 12-16), the minor bcr ( $\mathrm{m}-\mathrm{bcr}$; between exons 1-2), and the micro bcr ( $\mu$-bcr; between exons 17-20). More than $95 \%$ of CML cases and approximately half the cases with acute lymphoblastic leukemia with $\mathrm{t}(9 ; 22)$ have $B C R-A B L 1$ transcripts from the rearrangement at M-bcr, mostly the e14a2 (or b3a2) or e13a2 (or b2a2) types, producing a 210-kDa chimeric protein (P210). $B C R-A B L 1$ transcripts from $\mathrm{m}$-bcr produce a $190-\mathrm{kDa}$ chimeric protein (P190), with the ela2 type being the most common. The e1a2 type $B C R-A B L 1$ is frequently detected at low levels in CML with e14a2 or e13a2 type BCR-ABL1 [4]. However, e1a2 $B C R-A B L 1$ without other transcript types is rare in CML, accounting for approximately $1 \%$ of cases [5-9]. BCR-ABL1 transcripts (e19a2) from $\mu$-bcr produce a 230-kDa chimeric protein (P230) and are also rare. Interestingly, recent studies have reported that the type of $B C R-A B L 1$ transcripts affects the disease phenotype and outcome $[10,11]$. In particular, ela2 BCR-ABL1 have been reported to be associated with prominent monocytosis, minimal basophilia, poor response to TKI, higher rates of disease progression to accelerated/ blast phases, and poor survival [5-9]. In this report, we describe a patient who had prominent monocytosis mimicking chronic myelomonocytic leukemia (CMML) but was diagnosed as having CML after the genetic workup that demonstrated $\mathrm{t}(9 ; 22) / \mathrm{e} 1 \mathrm{a} 2 \mathrm{BCR}-A B L 1$ transcripts.

\section{CASE REPORT}

A 68-year-old woman was referred to the Division of Hematology-Oncology of Samsung Medical Center because of persistent thrombocytosis. She had been on follow-up for hypothyroidism. The complete blood counts (CBC) at referral showed leukocytosis and thrombocytosis with hemoglobin (Hb) $136 \mathrm{~g} / \mathrm{L}$, white blood cell (WBC) $19.470 \times 10^{9} / \mathrm{L}$, and platelets $737 \times 10^{9} / \mathrm{L}$ (Table 1 ). The differential counts of WBC were band neutrophils $1 \%$, neutrophils $56 \%$, eosinophils $1 \%$, basophils 4\%, lymphocytes 15\%, and monocytes 23\% (absolute monocyte count [AMC], $4.478 \times 10^{9} /$ L) (Fig. 1A). Neutrophils did not show definite dysplasia. The results from biochemical analysis were normal. Bone marrow (BM) study and related tests were performed with written informed consent from the patient and revealed hypercellular BM with $80 \%$ cellularity with increased granulopoiesis (without dysplasia) and megakaryopoiesis with dysplasia (megakaryocytes with separate nuclei and mononuclear forms) (Fig. 1B, C). Blasts were counted $4 \%$ on the aspirate smear. On the other hand, the CD34 immunostain on the biopsy section showed $5 \%$ to $10 \%$ CD34 ${ }^{+}$blasts (Fig. 1D). Conventional chromosome analysis using BM cells revealed $46, X X, t(9 ; 22)$ (q34.1;q11.2)(20) (Fig. 2A), and fluorescence in situ hybridization (FISH) using Vysis LSI BCR/ABL Dual Color, Dual Fusion Translocation Probe (Abbott Molecular, Des Plaines, IL, USA) showed $B C R$ and $A B L 1$ gene rearrangement in $99 \%$ of interphase nuclei: nuc ish 9q34.1 (ABL1x3), 22q11.2 (BCR×3) (ABL1 con BCRx2)(198/200) (Fig. 2B). Multiplex reverse transcriptase polymerase chain reaction (PCR) using HemaVision-28N (DNA Diagnostic, Risskov, Denmark) showed minor (P190) $B C R$-ABL1 transcripts of the ela2 type (Fig. 2C). Major (P210)

Table 1. Serial complete blood counts in the patient

\begin{tabular}{|c|c|c|c|c|c|c|}
\hline Parameter & Nov 11, 2015 & Nov 28, 2016 & Dec 6, 2017 & Dec 4, 2018 & $\operatorname{Jan} 18,2019^{a)}$ & Feb 28, 2019 \\
\hline Hemoglobin (g/L) & 141 & 136 & 125 & 124 & 135 & 106 \\
\hline $\mathrm{WBC}\left(\times 10^{9} / \mathrm{L}\right)$ & 5.380 & 6.94 & $9.830^{c)}$ & $14.770^{c)}$ & $19.470^{c)}$ & 2.59 \\
\hline Platelets $\left(\times 10^{9} / \mathrm{L}\right)$ & 236 & 250 & $386^{c)}$ & $602^{c)}$ & $737^{c)}$ & 79 \\
\hline $\operatorname{ANC}\left(\times 10^{9} / \mathrm{L}\right)$ & 2.948 & 3.69 & 5.71 & $8.860^{c)}$ & $10.903^{c)}$ & 1.04 \\
\hline $\mathrm{AMC}\left(\times 10^{9} / \mathrm{L}\right)$ & 0.462 & $1.048^{\mathrm{c})}$ & $2.035^{\mathrm{c})}$ & $2.363^{c)}$ & $4.478^{\mathrm{c})}$ & 285 \\
\hline Monocytes (\%) & $8.6^{c)}$ & $15.1^{\mathrm{c})}$ & $20.7^{c)}$ & $16^{c)}$ & $23^{c)}$ & 11 \\
\hline
\end{tabular}

Local reference ranges: $\mathrm{WBC}<8.630 \times 10^{9} / \mathrm{L}$, platelets $<347 \times 10^{9} / \mathrm{L}, \mathrm{ANC}<8.300 \times 10^{9} / \mathrm{L}, \mathrm{AMC}<0.690 \times 10^{9} / \mathrm{L}$, and monocytes $(\%)<8 \%$. WBC, white blood cell; ANC, absolute neutrophil count; AMC, absolute monocyte count.

a) At bone marrow study; ${ }^{\text {b) }}$ After 4 weeks of dasatinib medication; ${ }^{c}$ Leukocytosis, thrombocytosis, and monocytosis. 

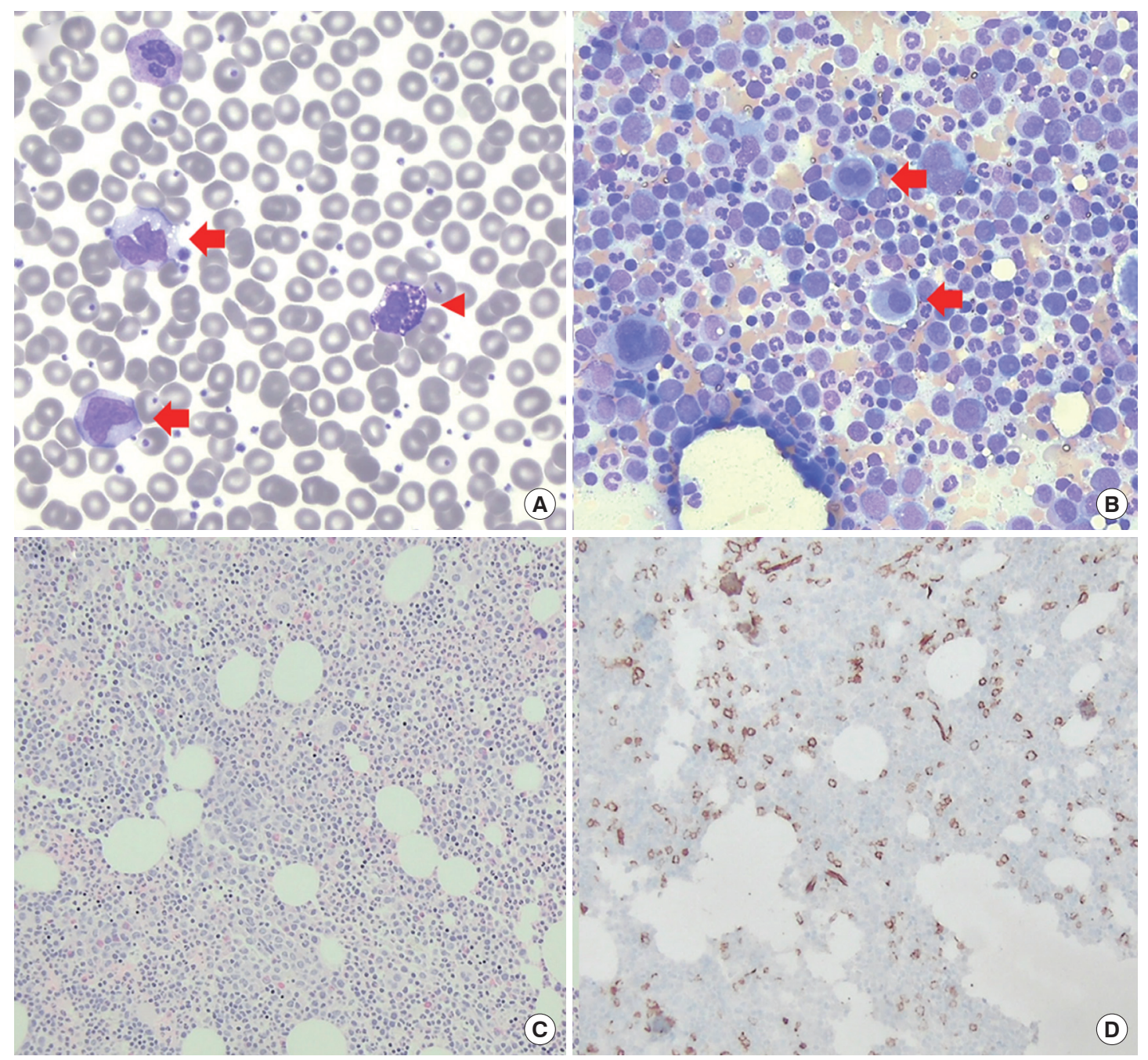

Fig. 1. (A) The peripheral blood smear of the patient showed leukocytosis with prominent monocytosis (red arrows), basophilia (red arrowhead) (white blood cell, $19.470 \times 10^{9} / \mathrm{L}$ with monocytes $23 \%$ and basophils $4 \%$ ), and thrombocytosis (platelets $737 \times 10^{9} / \mathrm{L}$; WrightGiemsa, $\times 1,000$ ). (B) Bone marrow study revealed increased granulocytic precursors and dysplastic megakaryocytes (red arrows) on aspirate smear (Wright-Giemsa, $\times 400$ ) and (C) increased cellularity (80\%) in biopsy section (H\&E stain, $\times 200)$. (D) CD34 immunostain on biopsy section showed increased CD34-positive cells $(>5 \% ; \times 200)$.

$B C R / A B L 1$ transcripts (including e14a2 or e13a2 types) or micro (P230) BCR-ABL1 transcripts (including e19a2) were not detected. Sanger sequencing of the $B C R-A B L 1$ transcripts in the patient confirmed the junction with in-frame fusion of exon 1 of $B C R$ and exon 2 of $A B L 1$, without additional nucleotide changes (primer sequences available upon request) (Fig. 2D). Molecular genetic tests for driver gene mutations in MPN (JAK2 V617F, JAK2 exon 12, MPL exon 10, and CALR exon 9 mutations) were performed using peripheral blood leukocytes and were all negative. Collectively, she was diagnosed with CML, BCR-ABL1-positive, in chronic phase. A review of serial $\mathrm{CBC}$ before the $\mathrm{BM}$ study demonstrated that isolated monocytosis preceded the abnormalities in other parameters of CBC (Table 1). The follow-up CBC after 4 weeks of da- satinib medication (100 mg daily) showed $\mathrm{Hb} 10^{6} \mathrm{~g} / \mathrm{L}$, WBC $2.590 \times 10^{9} / \mathrm{L}$, and platelets $79 \times 10^{9} / \mathrm{L}$.

\section{DISCUSSION}

According to the current World Health Organization classification of hematologic malignancies, there are three major disease categories in myeloid neoplasms other than acute myeloid leukemia: MPN, MPN/myelodysplastic syndrome (MDS), and MDS [1]. The MPN category includes CML (BCR-ABL1-positive), chronic neutrophilic leukemia (CNL), polycythemia vera (PV), and primary myelofibrosis (PMF). MPN/MDS includes CMML and atypical CML (BCR-ABL1-negative). The diagnostic criteria for each disease entity includes the presence 


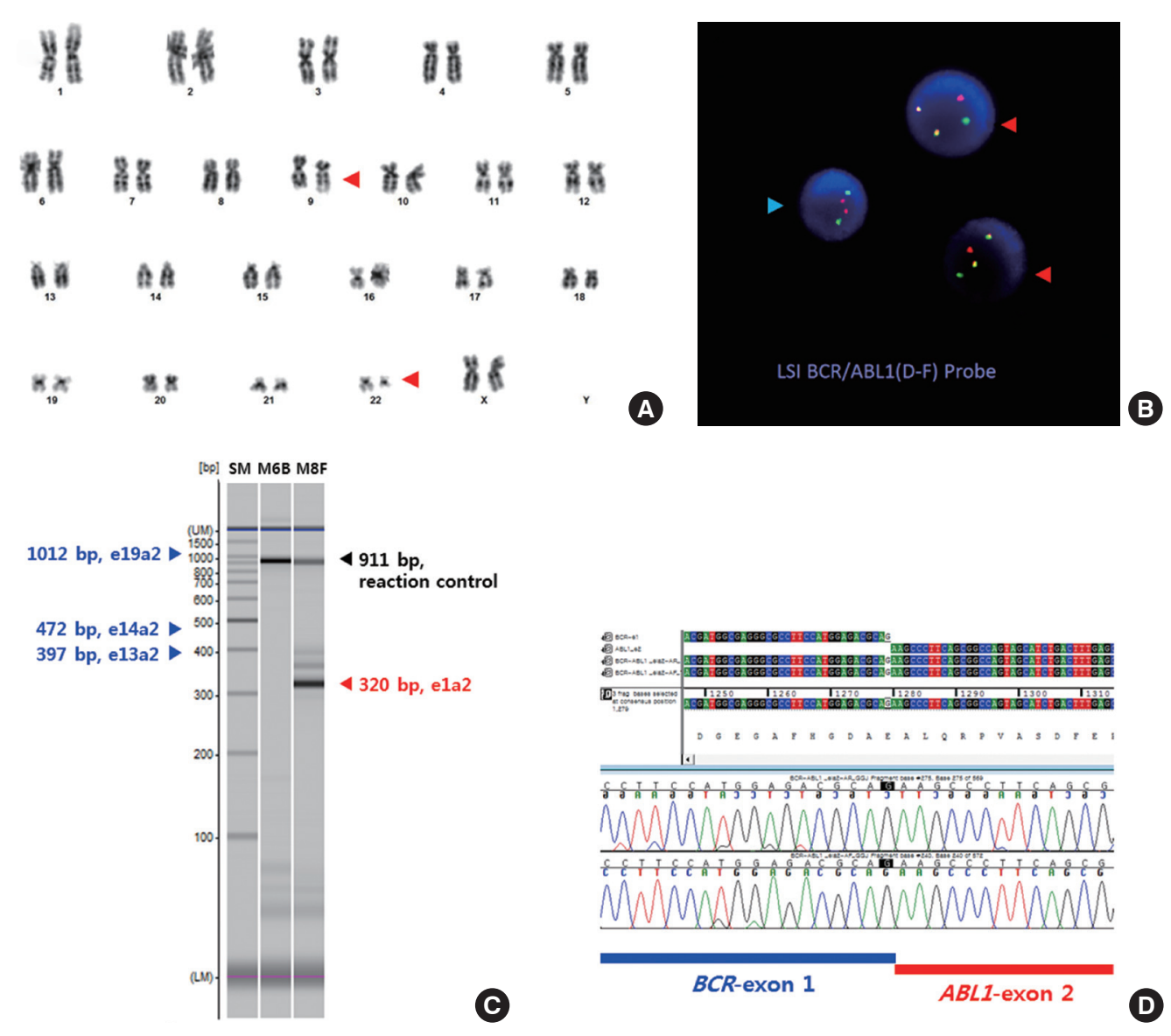

Fig. 2. (A) Conventional cytogenetic analysis revealed $t(9 ; 22)$ without additional cytogenetic aberrations (red arrows). (B) Fluorescence in situ hybridization study showed two fusion signals from $B C R-A B L 1$ rearrangement (red arrowheads) generated by rearrangement of the breakpoint cluster region $(B C R)$ gene on $22 q 11$ and the Abelson murine leukemia viral oncogene homolog 1 ( $A B L 1$ ) gene on $9 q 34$ in $99 \%$ interphase nuclei. Note the nucleus with two normal (non-rearranged) copies of $B C R$ and $A B L 1$ genes (two green and two red signals, respectively; lightblue arrowhead). (C) Multiplex reverse transcriptase polymerase chain reaction using the HemaVision kit revealed minor BCR-ABL1 fusion transcript (P190) with approximately 320 bp size, indicating e1a2 type, on the M8F panel (right lane, red arrowhead). No amplicons were detected in the middle lane (M6B) for major BCR-ABL1 transcripts, including e14a2 (b3a2) and e13a2 (b2a2) types (expected size 472 bp and $397 \mathrm{bp}$, respectively) and micro $B C R-A B L 1$, including e19a2 type (1,012 bp) (blue arrowheads). Left lane is for size marker (SM, 100-bp ladder), and bands at $911 \mathrm{bp}$ in the M6B and M8F lanes are reaction control amplicons (black arrowhead). (D) Sanger sequencing of the BCR-ABL1 transcripts confirmed in-frame fusion of the exon 1 of $B C R$ and exon 2 of $A B L 1$ without additional nucleotide changes.

of proliferative and/or dysplastic hematologic features (with predominantly affected lineages) and genetic aberrations. In particular, CMML is characterized by the presence of persistent ( $\geq 3$ months) peripheral blood monocytosis ( $\geq 1.000$ $\times 10^{9} / \mathrm{L}$ and $\geq 10 \%$ of WBC) and dysplasia involving $\geq 1$ myeloid lineages. While $t(9 ; 22) / B C R-A B L 1$ is required for the diagnosis of $\mathrm{CML}$, the absence thereof is a prerequisite for the diagnosis of the other disease entities including CMML.

The patient described in this report had persistent monocytosis with AMC $4.478 \times 10^{9} / \mathrm{L}$ ( $23 \%$ of WBC) along with thrombocytosis in peripheral blood without significant leftshifted neutrophils (1\%), suggesting the possibility of CMML (Table 1, Fig. 1A). However, the chromosome and FISH studies demonstrated $t(9 ; 22)(q 24 ; q 22)$ and $B C R-A B L 1$ rearrange- ment, respectively, indicating CML. Multiplex PCR revealed e1a2 type $B C R-A B L 1$ transcripts, which is very rare in CML. Of interest, the observation of this rare type of transcripts in this patient was in line with the manifestation with prominent monocytosis [5-9]. On the other hand, while CML with ela2 $B C R-A B L 1$ has been shown to be accompanied by minimal basophilia, our patient had apparently increased basophils at diagnosis $\left(7.788 \times 10^{9} / \mathrm{L}\right)$. It is also reported that $\mathrm{CML}$ with ela2 $B C R-A B L 1$ is associated with a high frequency of disease progression with increasing blasts. In our patient, the blast $\%$ on the aspirate smear was less than $5 \%$, but CD34 staining on the biopsy section revealed $>5 \% \mathrm{CD}^{4} 4^{+}$cells (Fig. 1D). The patient is currently under treatment with dasatinib, a 2ndgeneration TKI. Close monitoring is warranted, due to the as- 
sociation of ela2 $B C R-A B L 1$ with poor response to TKI therapy and rapid disease progression.

While CML with ela2 (P190) BCR-ABL1 is associated with prominent monocytosis and poor prognosis, $C M L$ with e19a2 (P230) BCR-ABL1 from the rearrangement involving $\mu$-bcr is associated with prominent neutrophilia, mimicking $\mathrm{CNL}$ (thus, is also referred to as neutrophilic CML), and with indolent course [12,13]. Pane et al. [12] suggested that the transcription silencer elements located in the intronic sequences within M-bcr of $B C R$ be the molecular basis of these different genotype-phenotype correlations [14]. Monocytosis also occurs in other MPN diseases. Barraco et al. [15] reported that $21 \%$ of patients with PV had monocytosis. Interestingly, they found significantly higher frequencies of tet methylcytosine dioxygenase 2 (TET2) and serine and arginine rich splicing factor 2 (SRSF2) gene mutations in PV with monocytosis than in PV without monocytosis. Since TET2 and SRSF2 are two of the most commonly mutated genes in CMML, this finding suggests that monocytosis in PV, and possibly CML, could be explained by CMML-associated mutations [16]. Monocytosis is also observed in PMF and was reported to be an independent predictor of inferior survival [17].

A review of the serial $C B C$ of the patient showed that the mild increase of monocyte percentage in the WBC differential counts $(8.6 \%)$ was the first flag to note (on November 30 , 2015), followed by AMC $\left(1.048 \times 10^{9} / \mathrm{L}, 15.1 \%\right)$ (on November $28,2016)$ (Table 1). Until then, Hb, WBC, and platelet counts were within the reference ranges. Leukocytosis and thrombocytosis were detected on December 6, 2017 and progressed until the referral for BM study on January 18,2019 . These findings suggest that the increase of monocytes might be the first hematopathologic change in CML with ela2 BCR-ABL1. Since the genetic workup for the diagnosis of MPN can be performed using WBC in peripheral blood without invasive BM study, suspicion of the disease from $C B C$ findings and timely genetic workup are important for early diagnosis and management.

In summary, we herein described a patient with CML from e1a2 (minor, P190) BCR-ABL1 transcripts, which is rare and associated with prominent monocytosis. Careful interpretation of $C B C$ and timely genetic workup to determine the precise type of $B C R-A B L 1$ transcripts are critical for the diagnosis and stratification of this rare subtype of CML with distinct genotype-phenotype correlations including poor prognosis.

\section{CONFLICTS OF INTEREST}

No potential conflict of interest relevant to this article was re- ported.

\section{ORCID}

Jae Won Yun https://orcid.org/0000-0002-9029-8036

Jung Yoon https://orcid.org/0000-0001-9296-5085

Jong Won Kim https://orcid.org/0000-0002-0708-9242

Sun-Hee Kim https://orcid.org/0000-0002-7542-5551

ChulWon Jung https://orcid.org/0000-0002-5474-6807

Hee-Jin Kim

https://orcid.org/0000-0003-3741-4613

\section{REFERENCES}

1. Swerdlow SH, editor. WHO classification of tumours of haematopoietic and lymphoid tissues. 4th ed. Lyon (FR): International Agency for Research on Cancer; 2017.

2. Jabbour E, Kantarjian H. Chronic myeloid leukemia: 2018 update on diagnosis, therapy and monitoring. Am J Hematol 2018;93:442-59.

3. Melo JV. The diversity of BCR-ABL fusion proteins and their relationship to leukemia phenotype. Blood 1996;88: 2375-84.

4. van Rhee F, Hochhaus A, Lin F, Melo JV, Goldman JM, Cross NC. p190 BCR-ABL mRNA is expressed at low levels in p210-positive chronic myeloid and acute lymphoblastic leukemias. Blood 1996;87:5213-7.

5. Verma D, Kantarjian HM, Jones D, Luthra R, Borthakur G, Verstovsek S, et al. Chronic myeloid leukemia (CML) with P190 BCR-ABL: analysis of characteristics, outcomes, and prognostic significance. Blood 2009;114:2232-5.

6. Arun AK, Senthamizhselvi A, Mani S, Vinodhini K, Janet NB, Lakshmi KM, et al. Frequency of rare BCR-ABL1 fusion transcripts in chronic myeloid leukemia patients. Int J Lab Hematol 2017;39:235-42.

7. Castagnetti F, Gugliotta G, Breccia M, lurlo A, Levato L, Albano $F$, et al. The BCR-ABL1 transcript type influences response and outcome in Philadelphia chromosome-positive chronic myeloid leukemia patients treated frontline with imatinib. Am J Hematol 2017;92:797-805.

8. Gong Z, Medeiros LJ, Cortes JE, Zheng L, Khoury JD, Wang W, et al. Clinical and prognostic significance of e1a2 BCR-ABL1 transcript subtype in chronic myeloid leukemia. Blood Cancer J 2017;7:e583.

9. Qin YZ, Jiang Q, Jiang H, Lai YY, Shi HX, Chen WM, et al. Prevalence and outcomes of uncommon BCR-ABL1 fusion transcripts in patients with chronic myeloid leukaemia: data from a single centre. Br J Haematol 2018;182:693- 
700.

10. Hanfstein B, Lauseker M, Hehlmann R, Saussele S, Erben $P$, Dietz $C$, et al. Distinct characteristics of e13a2 versus e14a2 BCR-ABL1 driven chronic myeloid leukemia under first-line therapy with imatinib. Haematologica 2014;99: 1441-7.

11. Ercaliskan A, Eskazan AE. The impact of BCR-ABL1 transcript type on tyrosine kinase inhibitor responses and outcomes in patients with chronic myeloid leukemia. Cancer 2018;124:3806-18.

12. Pane F, Frigeri F, Sindona M, Luciano L, Ferrara F, Cimino R, et al. Neutrophilic-chronic myeloid leukemia: a distinct disease with a specific molecular marker (BCR/ABL with C3/A2 junction). Blood 1996;88:2410-4.

13. Verstovsek S, Lin H, Kantarjian H, Saglio G, De Micheli D, Pane F, et al. Neutrophilic-chronic myeloid leukemia: low levels of p230 BCR/ABL mRNA and undetectable BCR/ABL protein may predict an indolent course. Cancer 2002; 94:2416-25.

14. Stewart MJ, Cox G, Reifel-Miller A, Kim SY, Westbrook CA, Leibowitz DS. A novel transcriptional suppressor located within a downstream intron of the BCR gene. J Biol Chem 1994;269:10820-9.

15. Barraco D, Cerquozzi S, Gangat N, Patnaik MM, Lasho T, Finke $\mathrm{C}$, et al. Monocytosis in polycythemia vera: clinical and molecular correlates. Am J Hematol 2017;92:640-5.

16. Itzykson R, Duchmann M, Lucas N, Solary E. CMML: clinical and molecular aspects. Int J Hematol 2017;105:711-9.

17. Tefferi A, Shah S, Mudireddy M, Lasho TL, Barraco D, Hanson CA, et al. Monocytosis is a powerful and independent predictor of inferior survival in primary myelofibrosis. $\mathrm{Br}$ J Haematol 2018;183:835-8. 